\title{
Effect of sodium bicarbonate ingestion upon repeated sprints
}

\author{
G. Lavender and S.R. Bird \\ Department of Movement Studies, Christ Church College, Canterbury, Kent, UK
}

\begin{abstract}
Summary
The purpose of the study was to assess the effect of sodium bicarbonate ingestion upon repeated bouts of intensive short duration exercise. Twenty-three subjects participated in the investigation (8 females and 15 males, age 21.4 2 2.3, mean $\pm s d)$. Subjects completed six trials; three following the ingestion of sodium bicarbonate $(300 \mathrm{mg} / \mathrm{kg}$ body weight) and three following the ingestion of a placebo $(8 \mathrm{~g}$ sodium chloride). Each trial consisted of ten ten-second sprints on a cycle ergometer with 50 seconds recovery between each sprint. 'Peak power' and 'average power output' during each ten second sprint was measured from the flywheel of the ergometer using a light-sensitive monitor (Cranlea) linked to a BBC microcomputer. The power outputs recorded during each ten-second sprint of the bicarbonate trials were then compared with those recorded during the corresponding sprint of the placebo trials. The bicarbonate trials produced higher mean 'average power' outputs in all ten of the ten-second sprints, with the difference in 'average power' output being statistically significant in eight of these $(p<0.05)$. The results also revealed that the difference in the 'average power' outputs attained during the bicarbonate and placebo trials increased as the number of sprint repetitions increased $(p<0.01)$. 'Peak power' output was also greater in the bicarbonate trials with it being significantly higher $(p<0.001)$ during the final ten-second sprint. It was concluded that during exercise consisting of repeated, short-duration sprints, power output was enhanced following the ingestion of sodium bicarbonate, $(300$ mg/kg body weight).
\end{abstract}

Keywords: Alkalosis, (repeated) sprints, lactic acid, sodium bicarbonate

\section{Introduction}

The fatigue experienced during intensive short-duration exercise has been attributed to lactacidosis ${ }^{1-8}$. The resulting fall in intramuscular $\mathrm{pH}$ is considered to inhibit muscular contraction in a number of ways: limiting the rate of resynthesis of ATP by inhibiting key glycolytic enzymes, in particular phosphofructokinase ${ }^{9,10}$; inhibiting the release of calcium ions from the sarcoplasmic reticulum and the binding of these ions to the protein troponin ${ }^{11,12}$; and impairing the neural impulse propagation ${ }^{13,14}$. It has therefore been suggested that if the fall in intramuscular $\mathrm{pH}$ could be delayed or prevented then anaerobic exercise performance would be enhanced.

Mainwood and Worsley-Brown ${ }^{15}$ have shown that the efflux of $\mathrm{H}^{+}$out of frog sartorious muscle could be accelerated by increasing the extracellular bicarbonate buffer. Recent studies have attempted to induce this effect by getting subjects to ingest sodium bicarbonate and then measuring the effects upon muscle metabolism and exercise performance. Some of these studies have shown a significant improvement in performance $e^{10,16-21}$ whilst others have not ${ }^{22-25}$.

Some studies have been criticized because the exercise duration has been inappropriate, with anaerobic metabolism only making a relatively small contribution to the energy requirements of the activity. Katz et $a l^{26}$. concluded that sodium bicarbonate did not help in sustained bouts of exercise but may help in repeated work bouts. It was the aim of this study to examine the effect of sodium bicarbonate ingestion upon repeated maximal sprints with a relatively short recovery between sprints.

\section{Materials and methods}

Eight females and 15 males (age 18-28) volunteered to act as subjects for this study. All were members of the movement studies department and thus fit and familiar with maximal exercise. In order to minimize changes in performance due to the subject familiarity with the trial protocol, all subjects undertook three preliminary familiarization sessions. These followed the exact procedures of the subsequent trials but subjects were given a placebo solution for these sessions. Subjects were not informed that these were familiarization sessions and therefore participated with full commitment.

In the investigation, subjects completed six trials; three following the ingestion of sodium bicarbonate ( $300 \mathrm{mg} / \mathrm{kg}$ body weight) and three following the ingestion of placebo ( $8 \mathrm{~g}$ sodium chloride), these being administered in a random order. A double blind design was used with test solutions allocated to subjects by an independent investigator. All test substances were dissolved in $400 \mathrm{ml}$ of a dilute blackcurrant drink to increase the palatability and partially disguise the slight difference in taste of the two substances. All test solutions were split into two equal doses of $200 \mathrm{ml}$, these being given one and two hours before the trial. Thus the bicarbonate dosages given to the subjects in 
Table 1. Illustrating the mean 'average power' values attained during the bicarbonate and placebo trials ( $n=23$ subjects, 3 bicarbonate trials and 3 placebo trials per subject). The standard deviations are for the whole group $(n=23)$

\begin{tabular}{|c|c|c|c|c|}
\hline \multicolumn{5}{|c|}{ Average power output (Watts) } \\
\hline \multirow{2}{*}{$\begin{array}{c}\text { Sprint } \\
\text { Repetition }\end{array}$} & \multirow{2}{*}{$\begin{array}{l}\text { Bicarbonate } \\
\text { mean SD }\end{array}$} & Placebo & \multirow[b]{2}{*}{ Difference } & \multirow[b]{2}{*}{ Significance } \\
\hline & & mean $S D$ & & \\
\hline 1 & $864.9 \pm 173.3$ & $857.9 \pm 158.4$ & 7.0 & n.s. \\
\hline 2 & $840.3 \pm 163.3$ & $832.7 \pm 143.7$ & 7.6 & $\mathrm{p}<0.05$ \\
\hline 3 & $811.9 \pm 153.5$ & $804.9 \pm 147.5$ & 7.0 & $p<0.05$ \\
\hline 4 & $790.9 \pm 148.7$ & $784.4 \pm 148.5$ & 6.5 & n.s. \\
\hline 6 & $762.0 \pm 138.1$ & $753.0 \pm 129.7$ & 9.0 & $\mathrm{p}<0.05$ \\
\hline 7 & $752.2 \pm 141.3$ & $741.5 \pm 130.2$ & 10.7 & $\mathrm{p}<0.01$ \\
\hline 8 & $747.4 \pm 141.8$ & $739.1 \pm 132.6$ & 8.3 & $\mathrm{p}<0.001$ \\
\hline 9 & $743.2 \pm 141.1$ & $732.3 \pm 132.1$ & 10.9 & $\mathrm{p}<0.01$ \\
\hline 10 & $745.4 \pm 143.1$ & $730.5 \pm 123.6$ & 14.9 & $\mathrm{p}<0.002$ \\
\hline
\end{tabular}

this investigation were the same as those used by others ${ }^{10,18,25}$.

Eight grams of table salt $(\mathrm{NaCl})$ was used as the placebo since it produced a similar taste to the sodium bicarbonate. Previous studies have used $\mathrm{NaCl}$ as a placebo in greater (Inbar et al. ${ }^{17}, 13$ grams) or lesser amounts (Costill et al. ${ }^{19}$ and Katz et al. ${ }^{26}, 1$ gram).

For the three hours prior to each trial, subjects were requested to refrain from eating, smoking or drinking anything other than water and the test solution. Subjects did not participate if they had been ill or had undertaken strenuous exercise within 24 hours of the trials. Prior to each trial the maximal nature of the exercise was stressed to the subjects. However, they were not informed of the aims of the study or of the use of a placebo in the investigation.

The cycle ergometer used in the trials was a Monark 864 with the seat adjusted to the appropriate height (the seat height was kept constant for each subject throughout all the trials). Prior to each trial subjects had a standard warm up which was a modified form of those used by Inbar $e t$ al $^{17}$ and Parry-Billings and MacLaren ${ }^{25}$. The warm up included three minutes cycling at $50 \mathrm{rpm}$ at a self selected resistance followed by a series of static stretching exercises. Subjects then pedalled for a further two minutes against half of the test load, as calculated from the formula for the Wingate Anaerobic Power test ${ }^{26}$. During these final two minutes, subjects completed two five second maximal sprints at 55 and 90 seconds. They then remained seated on the ergometer for a further three minutes before commencing the trial.

Each trial consisted of ten maximal sprints, each of ten seconds duration with 50 seconds recovery between each sprint. Subjects pedalled against a load calculated from the formula for the Wingate Anaerobic Power test ${ }^{26}$. A stationary start was used in accordance with the work of Coleman et al. who found it produced a higher peak power than a rolling start ${ }^{27}$. It also afforded a greater degree of consistency with the subjects beginning each sprint with the pedals in the same starting position. The power produced was measured from the flywheel using a light sensitive monitor (Cranlea) linked to a BBC microcomputer. This enabled the recording of the 'peak power' and the 'average power' output attained during each ten second sprint.

Subjects were given a five second verbal countdown to the start of each sprint, verbal encouragement throughout the sprint and verbal information on sprint duration. All trials were carried out in the physiological testing laboratory with only the subject and assessor present to prevent distractions. Data was analysed using analysis of variance and least significant difference tests. The power outputs attained for each sprint in the bicarbonate trials were compared with the power outputs attained during the corresponding sprint in the placebo trials.

In addition to the sodium bicarbonate and placebo trials, a subset of the group $(n=12)$ indertook two further sessions prior to which they ingested $400 \mathrm{ml}$ of the blackcurrant drink without the addition of sodium bicarbonate or sodium chloride. This was ingested in two $200 \mathrm{ml}$ doses one and two hours before the session, enabling assessment of any possible effect of the placebo $(\mathrm{NaCl})$ upon the subjects' power output.

\section{Results}

In a pilot study ten second sprints were shown to be appropriate in causing fatigue and in all trials there was a steady decline in 'peak power' and 'average power output' as the trial progressed from sprint repetition 1 to 10 . The work of Sutton et al. ${ }^{10}$ would suggest that fatigue experienced by subjects in this investigation could be attributed to lactic acid build up and the concurrent decrease in muscle $\mathrm{pH}$. The progressive decline in power output could be due to the 50 seconds passive recovery between each sprint being insufficient to allow a return to pre-exercise muscle $\mathrm{pH}$ and lactate levels ${ }^{28,29}$.

The results of the bicarbonate and placebo trials were compared using two-way analysis of variance tests-the data obtained from sprint 1 in the bicarbonate trials being compared with the data obtained for sprint 1 in the placebo trials etc. Thus a total of 20 analysis of variance tests were applied, ten for 'average power, (one for each sprint repetition) and ten for peak power' (one for each sprint repetition). Analysis of the 'average power' data revealed that for each sprint repetition a higher 'average power' was obtained in the bicarbonate trials, with the difference being statistically significant $(p<0.05)$ in eight of the ten sprints (Table 1 and Figure 1).

Further analysis of the data using a Spearman rank order correlation test revealed that the difference bet- 
Table 2. Illustrating the mean 'peak power' values attained during the bicarbonate and placebo trials ( $n=23$ subjects, 3 bicarbonate trials and 3 placebo trials per subject). The standard deviations are for the whole group $(n=23)$

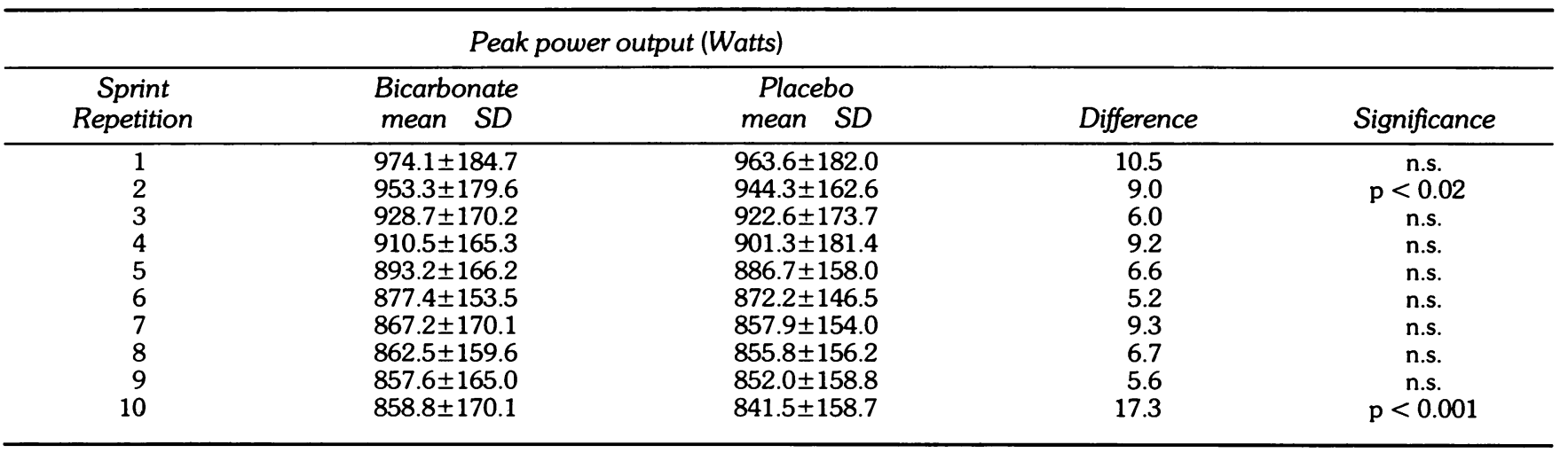

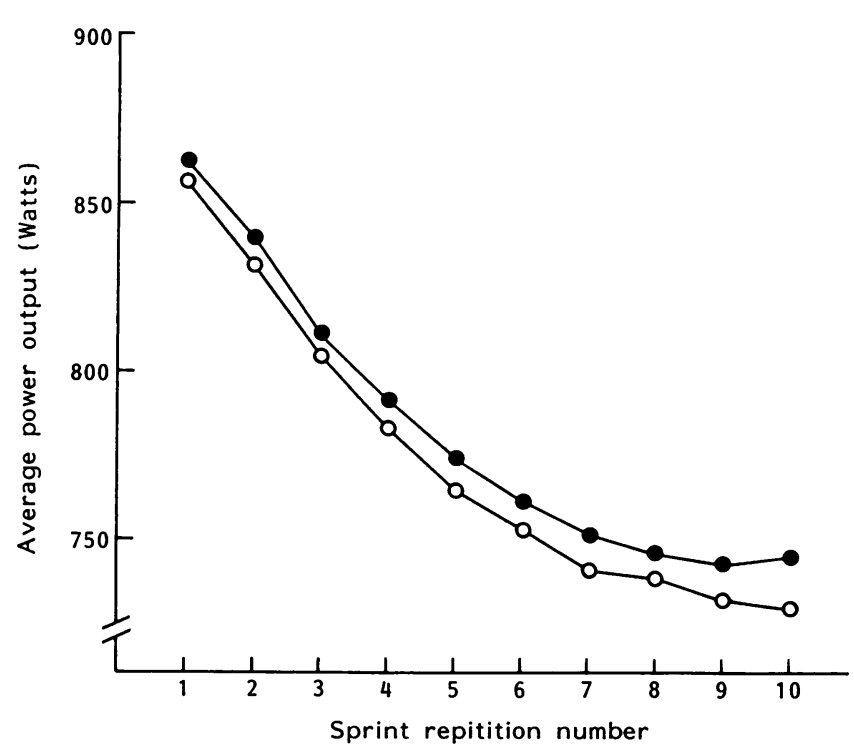

Figure 1. 'Average power' output for ten sprint repetitions (mean values for 23 subjects, 3 bicarbonate 1 and 3 placebo trials per subject)

ween the 'average power' output attained in the bicarbonate trials and placebo trials increased as the number of sprint repetitions increased $(p<0.01$, $r=0.821$ ).

A similar analysis conducted upon the 'peak power' data comparing each 'bicarbonate sprint repetition' with the corresponding 'placebo sprint repetition' revealed that higher 'peak power' values were attained in the bicarbonate trials. However, in only two of the sprint repetitions were these differences statistically significant (Table 2).

The results of the three placebo trials were then compared (two-way analysis of variance) with the results of the two sessions where only the blackcurrant (without the addition of sodium bicarbonate or placebo) had been drunk. The results showed that the placebo trials produced significantly higher 'average power' outputs in sprint repetitions 7 and $10(p<$ $0.05)$, and significantly higher 'peak power' outputs in repetitions 6,7 and $8(p<0.05)$.

\section{Discussions and conclusions}

A comparison between bicarbonate and placebo trials revealed that for each sprint repetition bicarbonate produced a higher 'average power' output, the difference being statistically significant in eight of the ten sprint repetitions. This observed difference could be attributed to the increased bicarbonate buffer ${ }^{21,25,28}$ accelerating the efflux of $\mathrm{H}^{+}$from muscle fibres, thereby reducing the fall in intramuscular $\mathrm{pH}^{10,17,18,30}$.

The data also revealed increasing differences between 'average power' outputs attained as the trials continued. This may be because the results from each trial indicate that the 50 seconds passive recovery between sprints was not sufficient to provide a complete recovery. This is likely to result in a progressive decrease in muscle $\mathrm{pH}$ and an accumulation of lactate as each trial progressed ${ }^{28,29}$.

However, in the bicarbonate trials the enhanced bicarbonate buffer would enable a greater efflux of $\mathrm{H}^{+}$ from the muscle thereby returning the muscle $\mathrm{pH}$ closer to its pre-exercise level than in the corresponding sprint of the placebo trial. This would therefore result in a more complete recovery between each sprint repetition during the bicarbonate trials. Therefore, the progressive fall in muscle $\mathrm{pH}$ that would occur during all trials would not only be reduced in the bicarbonate trials but would also occur at a slower rate. This would therefore result in a less acidic muscle $\mathrm{pH}$ at the start of each 'bicarbonate' sprint when compared to the muscle $\mathrm{pH}$ in the corresponding 'placebo' sprint, with the muscle $\mathrm{pH}$ in the placebo sprints becoming more acidic at a faster rate during each trial.

It would follow that in repeated short duration sprints, the effect of sodium bicarbonate ingestion would become more pronounced as the number of sprint repetitions increased. Indeed, Costill et al. ${ }^{19}$ suggested that single short duration sprints may be too short for the enhanced buffering capacity (induced by the ingestion of the sodium bicarbonate) to be effective, whereas longer duration sprints (1.75-2.0 minutes) might facilitate $\mathrm{H}^{+}$efflux from muscle increase effectiveness ${ }^{19}$. In this investigation, although the sprints were only of ten seconds duration, the repetitive nature of the exercise effectively made it an intermittent form of exercise lasting ten minutes. Therefore, the results observed appear to support the suggestions of 
Costill et al. confirming their suggestion that the duration of the exercise is important if the enhanced bicarbonate buffer is to be most effective.

Although the 'peak power' data indicated that the bicarbonate trials produced higher 'peak power' outputs than the placebo trials, the difference in 'peak power' output was only statistically significant in two of the sprint repetitions. Thus findings are in some agreement with Inbar et al. ${ }^{17}$ who found 'peak power' output unaffected by bicarbonate ingestion when assessed using a single 30 second sprint (Wingate Anaerobic Power test). However, data for the final sprint repetition in this investigation revealed a highly significant $(p<0.001)$ difference in 'peak power' output between the bicarbonate and placebo trials.

This makes it possible to speculate that the higher 'peak power' output attained in the bicarbonate trials was due to the increased bicarbonate buffer and psychological factors. It is suggested that during the final sprint repetition subjects were more highly motivated and that this factor coupled with the additional bicarbonate buffer resulted in a greater 'peak power' output being obtained. It is also possible to speculate that the partial conflict between the findings of this investigation and those of Inbar et al. ${ }^{17}$ were due to the repetitive nature of the sprints in this investigation compared with the single 30 second sprint used in their study.

When the results of the placebo trials were compared with those where only the blackcurrant was drunk (without the addition of sodium bicarbonate or placebo) small inconsistent differences in power output were observed. Where these results were statistically significant $(p<0.05)$ the power output was always higher in the placebo trials. It is difficult to suggest a physiological basis for these observed differences in power output and therefore it is suggested that they were primarily due to the ingestion of the placebo providing some psychological benefit to the subjects. The findings that the power outputs were not significantly lower in the placebo trials than in the trials where only blackcurrant was drunk enabled the investigators to conclude that the observed differences in power outputs between the placebo and bicarbonate trials were indeed due to an enhancement in power output during the bicarbonate trials, and not due to an impairment in power output during the placebo trials.

The results obtained in this study therefore support the findings of Sutton et al. ${ }^{10}$, Inbar et al. ${ }^{17}$, Wilkes et al. ${ }^{18}$, Costill et al. ${ }^{19}$, and Goldfinch et al. ${ }^{21}$, who concluded that the ingestion of sodium bicarbonate could improve performance in intensive short-duration exercise. In addition to this it can also be concluded that the ingestion of sodium bicarbonate can significantly increase power output during repeated maximal sprints with its effects becoming more pronounced as the number of repetitions increase.

\section{Acknowledgements}

The authors would like to thank Dr S. Bulleid for her technical assistance, Mr F.M. Holliday for the computer programmes used in the analysis of the data, and all the subjects who participated in the study.

\section{References}

1 Osnes, J.B. and Hermansen, L. Acid-base balance after maximal exercise of short duration J Appl Physiol 1972, $32,59-63$

2 Sahlin, K., Alvestrand, A., Brandt, R. and Hultman, E. Acid-base balance in blood during exhaustive bicycle exercise and the following recovery period Acta Physiol Scand 1978, 104, 370-372

3 Tesch, $P$. Muscle fatigue in man: with special reference to lactate accumulation during short term intense exercise Acta Physiol Scand Suppl 1980, 480

4 Jacobs, I., Bar-Or, O. Karlson, J., Dotan, R. and Tesch, $P$. Changes in muscle metabolites in females with $30 \mathrm{~s}$ exhaustive exercise Med Sci Sport Ex 1982, 14, 457-460

5 Jacobs, I., Tesch, P.A., Bar-Or, O., Karlson, J. and Dotan, R. Lactate in human skeletal muscle after 10s and 30s of supramaximal exercise J Appl Physiol 1983, $55,365-368$

6 Hultman, E. and Sjoholm, H. Energy metabolism and contraction force of human skeletal muscle in situ during electrical stimulation J Physiol 1983, 345, 525-532

7 Noble, B., Borg, G., Jacobs, I., Kaiser, P. and Cecci, R. Validation of a category-ratio perceived exertion scale: relationship to blood and muscle lactates and heart rate Med Sci Sports Ex 1983, 15, 523-528

8 Jacobs, I. Blood lactate: Implications for training and sports performance Sports Med 1986, 3, 10-25

9 Danforth, W.H. Activation of glycolytic pathway in muscle. In 'Control of energy metabolism' Eds. Chance, B., Estabrook, R.W. and Williamson, J.R. Academic Press, New York, 1965

10 Sutton, J.R., Jones, N.L. and Toews, C.J. Effect of $\mathrm{pH}$ on muscle glycolysis during exercise Clin Sci 1981, 61, 331-338

11 Donaldson, S.K.B. and Hermansen, L. Differential direct effects of $\mathrm{H}^{+}$and $\mathrm{Ca}^{2+}$-activated force of skinned fibres from the soleus, cardiac, adductor magnus muscles of rabbits Pflugers Arch 1978, 376, 55-65

12 Fabiato, A and Fabiato, F. Effects of $\mathrm{pH}$ on the myofilaments and sarcoplasmic reticulum of skinned cells from cardiac and skeletal muscles I Physiol 1978, 276, 233-235

13 Del Castillo, J., Nelson, T.E. Jr. and Sanchez, V. Mechanism of the increased acetylcholine sensitivity of skeletal muscle in low $\mathrm{pH}$ solutions J Cell Comp Physiol $1962,59,35-49$

14 Stephens, J.S. and Taylor, A. Fatigue of maintained voluntary muscle contraction in man J Physiol 1972, 22, $1-18$

15 Mainwood, G.W. and Worsley-Brown, P. The effects of extracellular $\mathrm{pH}$ and buffer concentration on the efflux of lactate from frog sartorius muscle $J$ Physiol $1975,250,1-22$

16 Jones, N.L., Sutton, J.R., Taylor, R. and Toews, C.J. Effect of $\mathrm{pH}$ on respiratory and metabolic responses to exercise J Appl Physiol 1977, 43, 959-964

17 Inbar, O., Rotstein, A., Jacobs, I., Kaiser, P., Dlin, R. and Dotan R. The effects of alkaline treatment on shortterm maximal exercise J Sports Sciences 1983, 1, 95-104

18 Wilkes, D., Gledhill, N. and Smyth, R. Effect of acute induced metabolic alkalosis on $800 \mathrm{~m}$ racing time Med Sci Sports Exerc 1983, 15(4), 277-280

19 Costill, D.L., Verstappen, F., Kuipers, H., Janssen, E. and Fink, W. Acid-base balance during repeated bouts of exercise: Influence of HCO Int J Sports Med 1984, 5, 228-231

20 MacLaren, D.P.M. and Morgan, G.D. Effects of sodium bicarbonate ingestion on maximal exercise Proc Nutr Soc 1985, 44, 26A

21 Goldfinch, J., McNaughton, L. and Davies, P. Induced metabolic acidosis and its effects on $400 \mathrm{~m}$ racing time Eur J Appl Physiol 1988, 57, 45-48 
Effect of sodium bicarbonate ingestion upon repeated sprints: G. Lavender et al.

22 Johnson, W.R. and Black, D.H. Comparison of effects of certain blood alkalinizers and glucose upon competitive endurance performance J Appl Physiol 1953, 5, 577578

23 Magaria, R., Aghemo, P. and Sassi, G. Effect of alkalosis on performance and lactate formation in supramaximal exercise Int Z Angew Physiol 1971, 29, 215223

24 Kindermann, W., Keul, J. and Huber, G. Physical exercise after induced alkalosis (bicarbonate or Tris-buffer) Eur I Appl Physiol 1977, 37, 197-204

25 Parry-Billings, M. and MacLaren, D.P.M. The effect of sodium bicarbonate and sodium citrate ingestion on anaerobic power during intermittent exercise Eur J Appl Physiol 1986, 55, 524-529

26 Dotan, R. and Bar-Or, O. Load optimization for the Wingate anerobic test Eur J Appl Physiol 1983, 51, 409417

26 Katz, A., Costill, D.L., King, D.S., Hargreaves, M. and
Fink, W.J. Maximal exercise tolerance after induced alkalosis Int J Sports Med 1984, 5, 107-110

27 Coleman, S.G.S., Hale, T. and Hamley, E.J. A comparison of power outputs with rolling and stationary starts in the Wingate anaerobic test J Sports Sci 1985, 3, 207

28 McCartney, N., Heigenhauser, G.J.F and Jones, N.L. Effects of $\mathrm{pH}$ on maximal power output and fatigue during short term dynamic exercise J Appl Physiol 1983, 55, 225-229

29 Rieu, M. Duvallet, A., Scharapan, L., Thieulart, L. and Ferry, A. Blood lactate accumulation in intermittent supramaximal exercise Eur J Appl Physiol 1988, 57, 235242

30 Mainwood, G.W. and Cechetto, D. The affect of bicarbonate concentration on fatigue and recovery in isolated rat diaphragm muscle Can J Physiol Pharmacol $1980,58,624-632$

The Dutch Medical Joggers Association is organizing the first international Medical Marathon in September 1989. They seek contact with all interested medical running groups to whom they will send full details of the event.

The Marathon Medical Symposium will be held on

Friday 15 September, and the full Marathon as well as a Half-Marathon Race and a $\mathbf{1 0} \mathbf{~ k m}$ Race will take place on Saturday 16 September 1989.

All races are open to running doctors, dentists, veterinarians and physiotherapists.

Venue: all events take place at the National Sports Centre, Papendahl, Arnhem, Netherlands. There is accommodation available at the centre itself.

Full details from: Dr Jacob A. Du Boeuff, President, Dutch Medical Joggers Association, PO Box 8153, NL-3503 RD, Utrecht Netherlands. 